Article

\title{
Design, Synthesis, and Biological Evaluation of Benzylamino- Methanone Based Cholesteryl Ester Transfer Protein Inhibitors
}

\author{
Ghassan Abu Sheikha, Reema Abu Khalaf *, Areej Melhem and Ghadeer Albadawi \\ Department of Pharmaceutical Sciences, Faculty of Pharmacy, Al-Zaytoonah Private University of \\ Jordan, Amman, Jordan; E-mail: pharmacy@alzaytoonah.edu.jo \\ * Author to whom correspondence should be addressed; E-Mail: \\ reema.abukhalaf@alzaytoonah.edu.jo or rima_abu_khalaf@yahoo.com; Tel.: +96264291511 ext. \\ 239; Fax: +962 64291511.
}

Received: 18 June 2010; in revised form: 10 August 2010 / Accepted: 13 August 2010 /

Published: 19 August 2010

\begin{abstract}
Cholesteryl ester transfer protein (CETP) is a glycoprotein involved in transporting lipoprotein particles and neutral lipids between high-density lipoprotein (HDL) and low density lipoproteins (LDL) and therefore its a proper target for treating dyslipidemia and related disorders. Guided by our previosuly-reported pharmacophore and QSAR models for CETP inhibition, we synthesized and bioassayed a series of benzylamino-methanones. The most potent illustrated 30\% CETP inhibition at $10 \mu \mathrm{M}$.
\end{abstract}

Keywords: CETP inhibitors; high-density lipoprotein; pharmacophore modeling; quantitative structure-activity relationship; benzylamino-methanones

\section{Introduction}

Atherosclerosis is the main cause for arterial dysfunction that limits blood flow to vessels in the peripheral vasculature and is finally marked as coronary artery disease (CAD) [1]. A number of epidemiological studies have established an inverse relationship between serum high-density lipoprotein (HDL) cholesterol levels and the incidence of ischemic heart disease [2]. HDL removes excess cholesterol from peripheral tissues to the liver for biliary elimination [3]. CETP, a 476-residue glycoprotein, is engaged in interchanging lipoprotein particles and neutral lipids, including cholesteryl esters, phospholipids and triglycerides between HDL and low density lipoproteins (LDL). CETP, as 
revealed by X-ray crystallography (PDB code: 2OBD, resolution $2.2 \AA$ ), has a huge highly lipophilic binding site capable of binding up to four lipid molecules [4]. In human plasma, CETP plays a probably proatherogenic task by moving cholesteryl esters from HDL to very-low density lipoprotein (VLDL) and LDL particles, thereby lowering atheroprotective HDL cholesterol and raising proatherogenic VLDL and LDL cholesterols. Obviously, the risk of CAD is proportional to the plasma levels of CETP [5]. Actually, It is rather frequent within the CAD population to have elevated CETP plasma protein levels that are 2- to 3-fold higher than concentrations typically found in the plasma of normal subjects $(1-3 \mu \mathrm{g} / \mathrm{mL})[6]$.

Indication exists that the outcomes of CETP activity may depend on the metabolic setting, particularly on triglyceride levels. Therefore, pharmacological CETP inhibition may reduce the risk of CAD in humans, but only in those with high triglyceride levels [5].

The inaccessibility of a reasonable high resolution crystallographic structure for CETP combined with its large binding pocket locked up most modeling-related discovery projects to ligand-based approaches particularly quantitative structure-activity relationship analysis (QSAR) [7-11]. Earlier, we have developed ligand-based three-dimensional (3D) pharmacophores integrated within a selfconsistent QSAR model for CETP inhibitors. The pharmacophore models were used as 3D search queries to mine 3D libraries for new CETP inhibitors, while the QSAR model predicted their biological activities and therefore prioritize them for in vitro evaluation [12]. Additionally, we recently described the synthesis of several benzylidene-amino methanones, (including 1, Figure 1), as representatives of a new series of simple CETP inhibitors [12]. Herein we describe our efforts to optimize the activity of this series of compounds through reduction of the imine double bond to the corresponding amine analogues (compounds 19-30, Scheme 2).

Figure 1. The structure of benzylidene-amino methanone derivative $\mathbf{1}$.

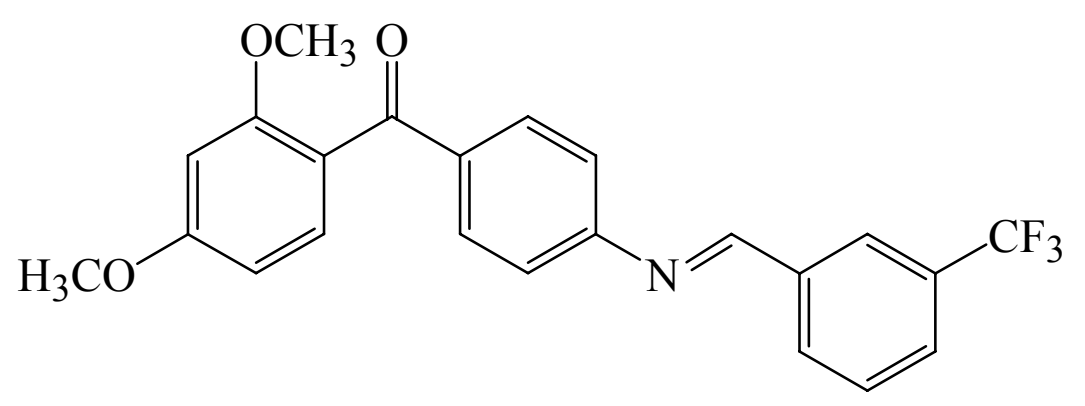

The new compounds are expected to have better anti-CETP bioactivities compared to the previously synthesized rigid benzylidene-amino methanones due to the enhanced flexibility of the amino analogues, which should allow better fit values against the pharmacophores i.e. to fit both QSARemerged pharmacophores instead of mapping just one of them. Furthermore, amino-derivatives are more stable than imines in aqueous conditions [13]. On the other hand, reduction of imines into amines can alter their physicochemical properties such as lipophilicity and basicity of the nitrogen, whereby the amines are more water soluble and more basic than their imine analogues. This property modification can influence the anti-CETP activity of the synthesized compounds. The synthesis commenced by preparing different substituted imine intermediates 8-11 (Scheme 1). Imines are 
typically formed by reversible acid-catalyzed condensation of amines and aldehydes with extrusion of water through either azeotropic distillation or by employing chemical drying agents [14].

Scheme 1. Synthesis of 4-aminobenzoic acid derivatives 12-15.

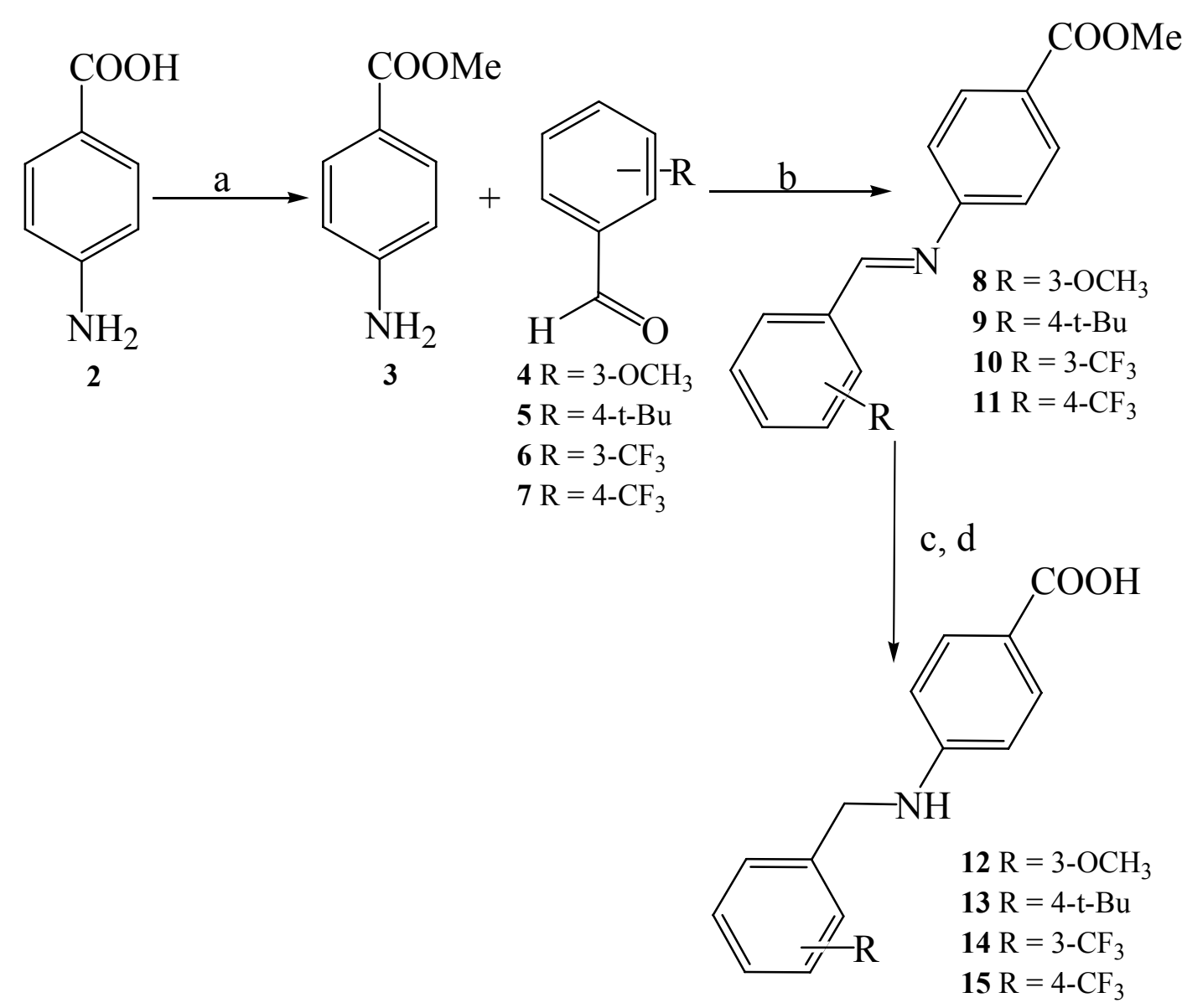

Reagents and conditions: (a) Methanol, $\mathrm{SOCl}_{2}$; (b) DMF, 90-100 ${ }^{\circ} \mathrm{C}$, overnight; (c) $\mathrm{NaBH}_{4}$, methanol, overnight; (d) $\mathrm{NaOH}, 100{ }^{\circ} \mathrm{C}$, overnight.

Subsequently, the imine intermediates were reduced to their corresponding amines 12-15 (Scheme 1) which were then used to prepare the final benzylamino-methanones, in modest to reasonable yields, via Friedel-Crafts acylation of the substituted benzene derivatives 16-18 in the presence of polyphosphoric acid (PPA), as reported in Scheme 2 [15]. The structures proposed for compounds 1930 were confirmed via elemental analyses, IR and ${ }^{1} \mathrm{H}$ - and ${ }^{13} \mathrm{C}-\mathrm{NMR}$ spectroscopy (see the Experimental section). 
Scheme 2. Synthesis of benzylamino-methanones 19-30.

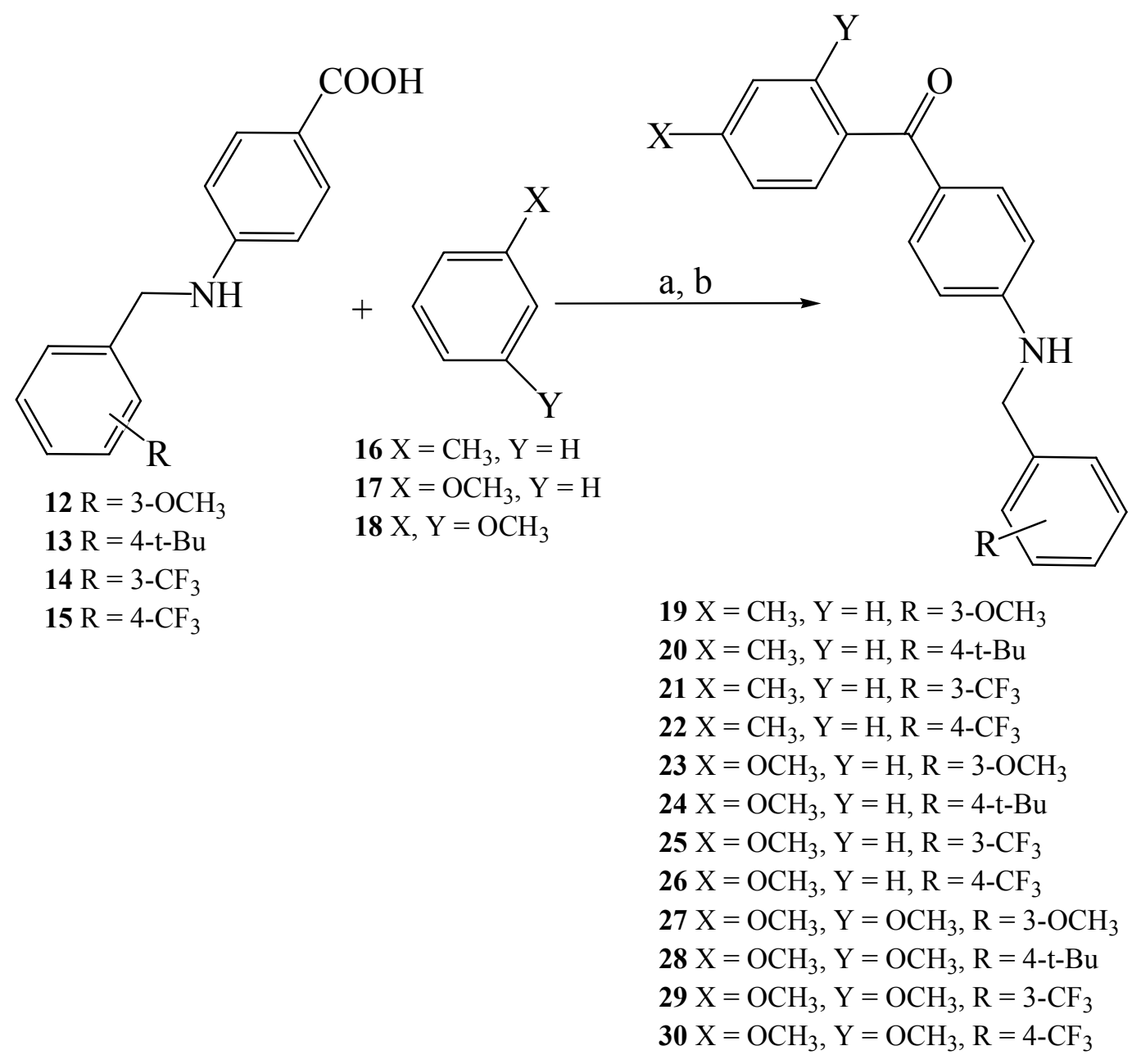

Reagents and conditions: (a) PPA, cyclohexane, $90-100{ }^{\circ} \mathrm{C}$, overnight; (b) $\mathrm{NH}_{3}, 0{ }^{\circ} \mathrm{C}$.

\section{Results and Discussion}

In the current work, the imine intermediates were prepared from reaction of trifluoro- $m$ tolualdehyde, trifluoro- $p$-tolualdehyde, 3-methoxybenzaldehyde and 4-tert-butylbenzaldehyde with the methyl ester of 4- aminobenzoic acid (3) as illustrated in Scheme 1 [16]. The best yield was obtained when 3, dissolved in DMF, reacted with trifluoro- $p$-tolualdehyde to yield $\mathbf{1 5}$ (92.2\%). Afterward, the imine intermediates were reduced to their corresponding amines, which were then used to prepare the final benzylamino-methanones. The best yield was obtained upon reacting 4-aminobenzoic acid derivative 15 with toluene to yield 22 (96\%). Scheme 2 shows the new benzylamino-methanone derivatives 19-30, while Figure 2 shows how Hypo4/8 and Hypo12/4 map compounds 26 and 27. 
Figure 2. The binding pharmacophore hypotheses emerged in the optimal QSAR model (Hydrogen bond acceptor as green vectored spheres, hydrophobic features as blue spheres, ring aromatic as orange vectored spheres, Hydrogen bond donor as violet vectored spheres): (A) Hypo4/8 mapping 26, (B) Hypo4/8 mapping 27, (C) Hypo12/4 mapping 26, (D) Hypo12/4 mapping 27, (E) and (F) The chemical structures of $\mathbf{2 6}$ and 27, respectively.

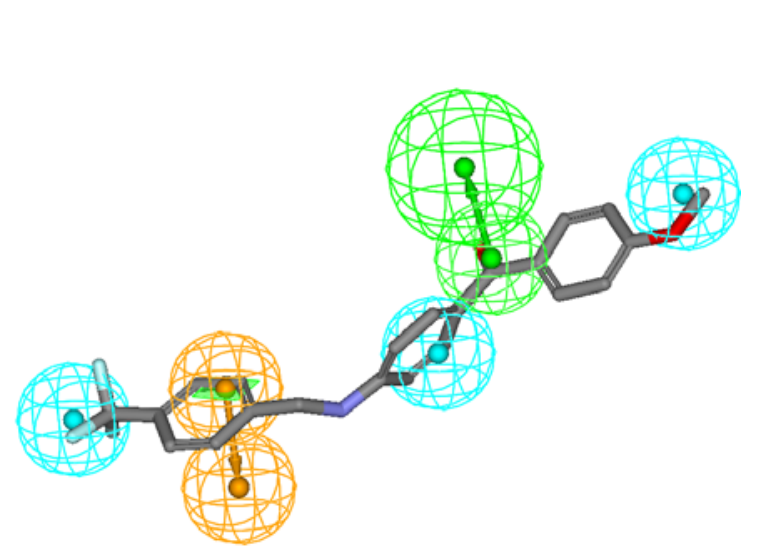

(A)

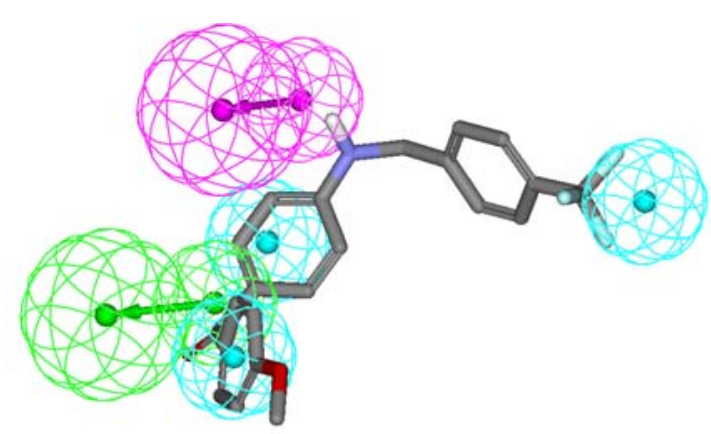

(C)<smiles>COc1ccc(C(=O)c2ccc(NCc3ccc(C(F)(F)F)cc3)cc2)cc1</smiles>

(E)

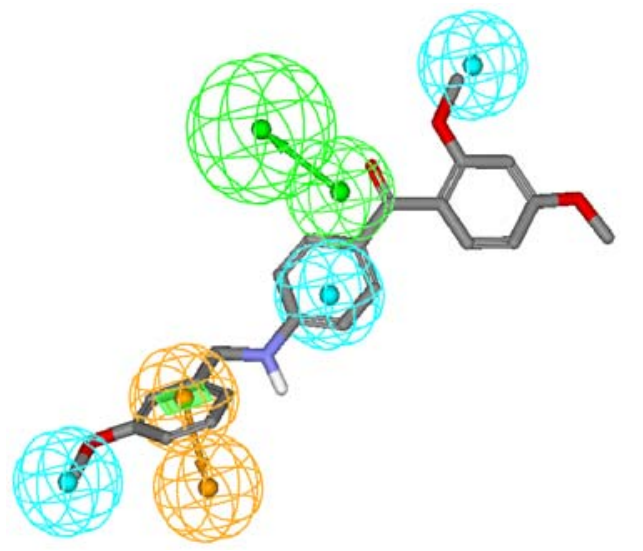

(B)

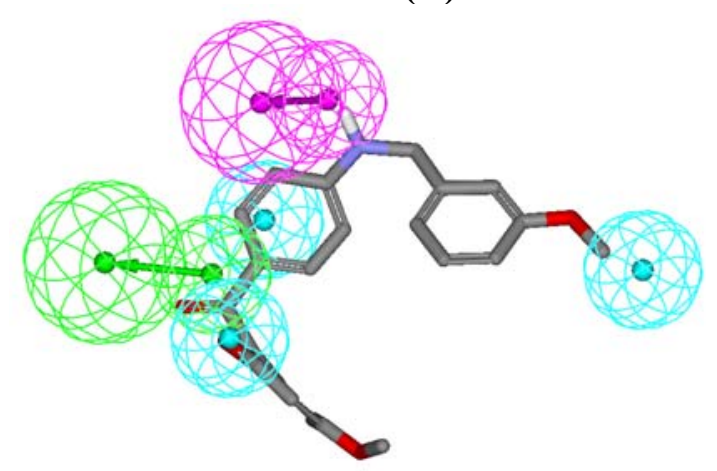

(D)<smiles>COc1cccc(CNc2ccc(C(=O)c3ccc(OC)cc3OC)cc2)c1</smiles>

(F)

As can be noticed from Table 1, the benzylamino-methanone compounds have enhanced flexibility reflected by their fit values against Hypo12/4 in comparison to their benzylidene-amino methanones which do not fit Hypo12/4.

In this work, we intended to make the compounds more flexible in order to enhance their pharmacophore mapping in an attempt to increase their anti-CETP bioactivities, but it seems that the entropic cost contribution overcomes the enhanced flexibility contribution to the bioactivity. Therefore, pharmacophore fitness is just one of many factors that can influence the activity.

The results of anti-CETP activity tests, presented in Table 1, demonstrate that compound 26 exhibited appreciable activity against CETP. Although our newly synthesized benzylamino- 
methanones are of lower potency than some published CETP inhibitors, these derivatives are characterized by their novel scaffold that could be a promising lead for further optimization.

The new compounds 19-30 were synthesized to explore how the CETP inhibitory activity is affected by the expansion of the structure, i.e. meta or para substitution, and the electronic properties of the substituent, i.e. electron donating or with-drawing group.

Table 1. The synthesized benzylamino-methanones with their fit values, corresponding QSAR estimates and in vitro bioactivities.

\begin{tabular}{|c|c|c|c|c|c|c|}
\hline \multirow{2}{*}{ Compound } & \multicolumn{2}{|c|}{ Fit values against } & \multicolumn{2}{|c|}{$\begin{array}{c}\text { QSAR-based } \\
\text { estimates }\end{array}$} & \multirow{2}{*}{$\begin{array}{c}\text { In vitro \% } \\
\text { inhibition of } \\
\text { CETP at } 10 \mu \mathrm{M}\end{array}$} & \multirow{2}{*}{$\begin{array}{c}\text { In vitro } \\
\text { IC }_{50}(\mu \mathrm{M})\end{array}$} \\
\hline & Нуро4/8 & Нуро12/4 & $\log \left(1 / I C_{50}\right)$ & $\begin{array}{l}\mathrm{IC}_{50} \\
(\mu \mathrm{M})\end{array}$ & & \\
\hline 19 & 7.9 & 2.2 & -0.936 & 8.6 & $16.4 \pm 3.0$ & - \\
\hline 20 & 8.3 & 0.8 & -1.25 & 17.8 & $21.6 \pm 0.8$ & $\begin{array}{c}60.3 \\
(0.99)^{\mathrm{a}}\end{array}$ \\
\hline 21 & 6.5 & 1 & -1.282 & 19.1 & $16.7 \pm 0.6$ & - \\
\hline 22 & 7.6 & 1.3 & -0.86 & 7.2 & $12.6 \pm 2.6$ & - \\
\hline 23 & 8.6 & 2 & -1.111 & 12.9 & $20.0 \pm 3.2$ & $\begin{array}{c}66.1 \\
(0.99)^{\mathrm{a}}\end{array}$ \\
\hline 24 & 8.3 & 1.8 & -1.289 & 19.5 & $23.3 \pm 2.3$ & $\begin{array}{c}51.3 \\
(1.00)^{\mathrm{a}}\end{array}$ \\
\hline 25 & 8.2 & 0.3 & -1.051 & 11.2 & $9.8 \pm 0.4$ & - \\
\hline 26 & 7.8 & 2.3 & -0.754 & 5.7 & $29.9 \pm 2.8$ & $\begin{array}{c}25.1 \\
(0.99)^{\mathrm{a}}\end{array}$ \\
\hline 27 & 9.4 & 3.1 & -0.954 & 9 & $24.3 \pm 2.7$ & $\begin{array}{c}36.3 \\
(0.99)^{\mathrm{a}}\end{array}$ \\
\hline 28 & 9.4 & 2.8 & -0.823 & 6.7 & $21.3 \pm 2.5$ & $\begin{array}{c}61.7 \\
(0.99)^{\mathrm{a}}\end{array}$ \\
\hline 29 & 8.5 & 1.4 & -1.314 & 20.6 & $19.5 \pm 0.6$ & - \\
\hline 30 & 8.8 & 2.1 & -1.089 & 12.3 & $19.8 \pm 2.9$ & - \\
\hline
\end{tabular}

${ }^{\mathrm{a}}$ This value represents the correlation coefficient of the corresponding dose-response line at three concentrations.

As a general trend, the inhibitory activity of compound 26, that is para-substituted (R) with a trifluoromethyl electron withdrawing group and fully extended, decreases with the trifluoromethyl group on the meta position as in compound 25. Furthermore, meta and para substitution (R) with electron donating groups such as $t$-Bu in compounds $\mathbf{2 0}$ and $\mathbf{2 4}$, and methoxy in compound $\mathbf{2 3}$ seems to conserve the anti-CETP activity. Moreover, mono- $(\mathrm{X})$ or di-substitution (X and Y) of the aromatic ring, as in compounds $\mathbf{2 4}$ and $\mathbf{2 8}$, respectively, appears not to have a considerable effect on the inhibitory activity.

Compounds 19-30 were tested against CETP at $10 \mu \mathrm{M}$ concentrations and exhibited anti-CETP activity ranging from 9.8 to $29.9 \%$. Compound $\mathbf{2 6}$ displayed the best activity as reported in Table 1 . Furthermore, in vitro $\mathrm{IC}_{50}$ values were determined for the most active compounds and approximately 3 to 9 fold differences were observed between QSAR-based $\mathrm{IC}_{50}$ estimates and the experimental $\mathrm{IC}_{50}$ values. 


\section{Conclusions}

In conclusion, we have successfully achieved synthetic exploration of a new series of aromatic amines as CETP inhibitors. They showed comparable activities to their benzylidene-amino methanones analogues [12] which reveals that flexibility of these amines was not enough to influence their antiCETP activity. We are currently in the process of preparing new compounds of better bioactivity profiles.

\section{Experimental}

\section{General methods}

Melting points were measured using Gallenkampf melting point apparatus and are uncorrected. ${ }^{1} \mathrm{H}$ - NMR and ${ }^{13} \mathrm{C}$-NMR spectra were collected on a Varian Oxford $\mathrm{NMR}^{300}$ spectrometer. The samples were dissolved in $\mathrm{CDCl}_{3}$. Mass spectrometry was performed using LC Mass Bruker Apex-IV mass spectrometer utilizing an electrospray interface. Infrared spectra were recorded using Shimadzu IRAffinity-1 spectrophotometer. The samples were dissolved in $\mathrm{CHCl}_{3}$ and analysed as thin solid films using $\mathrm{NaCl}$ plates. Analytical thin layer chromatography (TLC) was carried out using pre-coated aluminum plates and visualized by UV light (at 254 and/ or $360 \mathrm{~nm}$ ). Elemental analysis was performed using EuroVector elemental analyzer. Chemicals and solvents were purchased from the corresponding companies (Sigma-Aldrich, Riedel-de Haen, Fluka, BDH Laboratory Supplies and Promega Corporation) and were used in the experimentation without further purification.

\section{General procedure for the synthesis of 4-aminobenzoic acid derivatives 12-15}

4-Aminobenzoic acid $(2,1.37 \mathrm{~g}, 10 \mathrm{mmol})$ was dissolved in methanol $(100 \mathrm{~mL})$, and then thionyl chloride $(200 \mathrm{mmol})$ was added at $0{ }^{\circ} \mathrm{C}$. The mixture was stirred at room temperature for $20-30$ minutes, followed by refluxing at $65-70{ }^{\circ} \mathrm{C}$ overnight. Evaporation of the solvent was carried out, followed by neutralization using $\mathrm{K}_{2} \mathrm{CO}_{3}$ and extraction with $\mathrm{CH}_{2} \mathrm{Cl}_{2}(3 \times 20 \mathrm{~mL})$. The combined extracts were dried on anhydrous $\mathrm{Na}_{2} \mathrm{SO}_{4}$ and filtrated to give 4-amino-benzoic acid methyl ester (3, 96\%). Subsequently, 3 (1.52 g, $10 \mathrm{mmol})$ was disolved in DMF $(10 \mathrm{~mL})$, then an aldehyde (4-7, 25 mmol) was added. The mixture was heated between $100-150{ }^{\circ} \mathrm{C}$ overnight. After removing DMF, methanol $(15 \mathrm{~mL})$ was added to the reaction mixture, followed by gradual addition of $\mathrm{NaBH}_{4}(4$ equivalents) and stirring at room temperature overnight. The residue, after evaporation of the solvent, was purified by column chromatography eluting with cyclohexane/EtOAc (90:10). Next, desterification was carried out by refluxing with $1 \mathrm{M} \mathrm{NaOH}\left(2.6\right.$ equivalents) at $100{ }^{\circ} \mathrm{C}$ overnight. Then, the reaction mixture was neutralized with $\mathrm{HCl}$ and extracted with $\mathrm{CHCl}_{3}(3 \times 20 \mathrm{~mL})$. The combined extracts were dried on anhydrous $\mathrm{Na}_{2} \mathrm{SO}_{4}$ and filtered.

4-(3-Methoxybenzylamino)-benzoic acid (12). Evaporation of the solvent gave $\mathbf{1 2}$ as an off-white powder (88\%); mp. $160-161{ }^{\circ} \mathrm{C}$; ${ }^{1} \mathrm{H}-\mathrm{NMR}\left(300 \mathrm{MHz}, \mathrm{CDCl}_{3}\right) \delta 3.68(\mathrm{~s}, 3 \mathrm{H}), 4.26(\mathrm{~s}, 2 \mathrm{H}), 4.72$ (br s, $1 \mathrm{H}), 6.55(\mathrm{~d}, J=8.8 \mathrm{~Hz}, 2 \mathrm{H}), 6.77(\mathrm{dd}, J=8.1,1.4 \mathrm{~Hz}, 1 \mathrm{H}), 6.86-6.89(\mathrm{~m}, 2 \mathrm{H}), 7.21(\mathrm{t}, J=8.1 \mathrm{~Hz}$, $1 \mathrm{H}), 7.61(\mathrm{~d}, J=8.8 \mathrm{~Hz}, 2 \mathrm{H}), 12.03(\mathrm{br} \mathrm{s}, 1 \mathrm{H}) ;{ }^{13} \mathrm{C}-\mathrm{NMR}\left(300 \mathrm{MHz}, \mathrm{CDCl}_{3}\right) \delta 46.3(1 \mathrm{C}), 55.4(1 \mathrm{C})$, 111.7 (1C), 112.5 (1C), 113.3 (1C), 117.6 (1C), 119.8 (1C), 130.0 (2C), $131.6(2 \mathrm{C}), 141.7$ (1C), 152.9 
(1C), 159.9 (1C), 168.0 (1C); IR (thin film) $\mathrm{cm}^{-1}$ 3437, 3059, 2940, 1655, 1597, 1489, 1427, $1285,1177$.

4-(4-tert-Butylbenzylamino)-benzoic acid (13). Evaporation of the solvent gave $\mathbf{1 3}$ as an off-white powder (83\%); mp. $210-211{ }^{\circ} \mathrm{C}$; ${ }^{1} \mathrm{H}-\mathrm{NMR}\left(300 \mathrm{MHz}, \mathrm{CDCl}_{3}\right) \delta 1.27$ (s, 9H), $4.36(\mathrm{~s}, 2 \mathrm{H}), 4.80$ (br s, $1 \mathrm{H}), 6.60(\mathrm{~d}, J=8.7 \mathrm{~Hz}, 2 \mathrm{H}), 7.26-7.36(\mathrm{~m}, 4 \mathrm{H}), 7.94(\mathrm{~d}, J=8.7 \mathrm{~Hz}, 2 \mathrm{H}), 11.87(\mathrm{br} \mathrm{s}, 1 \mathrm{H}) ;{ }^{13} \mathrm{C}-\mathrm{NMR}$ $\left(300 \mathrm{MHz} \mathrm{CDCl}_{3}\right) \delta 31.4(3 \mathrm{C}), 34.6(1 \mathrm{C}), 47.4(1 \mathrm{C}), 111.6(2 \mathrm{C}), 117.6(1 \mathrm{C}), 125.8(2 \mathrm{C}), 127.4(2 \mathrm{C})$, 132.4 (2C), 135.2 (1C), 150.7 (1C), 152.5 (1C), 172.4 (1C); IR (thin film) $\mathrm{cm}^{-1} 3426,3017,2963$, 1670, 1605, 1524, 1478, 1420, 1292.

4-(3-Trifluoromethylbenzylamino)-benzoic acid (14). Evaporation of the solvent gave $\mathbf{1 4}$ as an offwhite powder (77\%); mp. 168-169 ${ }^{\circ} \mathrm{C} ;{ }^{1} \mathrm{H}-\mathrm{NMR}\left(300 \mathrm{MHz}, \mathrm{CDCl}_{3}\right) \delta 4.41(\mathrm{~s}, 2 \mathrm{H}), 4.77$ (br s, 1H), $6.57(\mathrm{~d}, J=8.6 \mathrm{~Hz}, 2 \mathrm{H}), 7.53-7.66(\mathrm{~m}, 6 \mathrm{H}), 11.93($ br s, $1 \mathrm{H}) ;{ }^{13} \mathrm{C}-\mathrm{NMR}\left(300 \mathrm{MHz}, \mathrm{CDCl}_{3}\right) \delta 45.7$ (1C), 111.7 (2C), 118.0 (1C), 124.0 (1C), 124.1 (1C), 127.6 (1C), 129.9 (1C), 131.6 (2C), 131.8 (2C), 141.7 (1C), 152.6 (1C), 167.9 (1C); IR (thin film) $\mathrm{cm}^{-1} 3456,3062,2920,1674,1605,1516,1481$, $1424,1316,1107$.

4-(4-Trifluoromethylbenzylamino)-benzoic acid (15). Evaporation of the solvent gave $\mathbf{1 5}$ as a pale yellow powder (93\%); mp. $189-190{ }^{\circ} \mathrm{C}$; ${ }^{1} \mathrm{H}-\mathrm{NMR}\left(300 \mathrm{MHz}, \mathrm{CDCl}_{3}\right) \delta 4.41$ (s, 2H), 4.79 (br s, $\left.1 \mathrm{H}\right)$, $6.54(\mathrm{~d}, J=8.8 \mathrm{~Hz}, 2 \mathrm{H}), 7.51(\mathrm{~d}, J=8.1 \mathrm{~Hz}, 2 \mathrm{H}), 7.59-7.67(\mathrm{~m}, 4 \mathrm{H}), 12.02(\mathrm{br} \mathrm{s}, 1 \mathrm{H}) ;{ }^{13} \mathrm{C}-\mathrm{NMR}(300$ $\left.\mathrm{MHz}, \mathrm{CDCl}_{3}\right) \delta 45.8(1 \mathrm{C}), 111.7$ (2C), 118.0 (1C), 123.1 (1C), 125.8 (2C), 127.8 (1C), 128.2 (2C), 131.6 (2C), 145.2 (1C), 152.6 (1C), 167.9 (1C); IR (thin film) $\mathrm{cm}^{-1} 3414,3042,2920,1659,1605$, $1520,1462,1424,1323,1122$.

\section{General procedure for the synthesis of benzylamino-methanones 19-30}

4-Aminobenzoic acid derivative 12-15 (2 mmol) was dissolved in cyclohexane (10 $\mathrm{mL})$, and polyphosphoric acid (6.5 g) was added. Then a benzene derivative 16-18 $(20 \mathrm{mmol})$ was added. The mixture was stirred carefully at $90-110{ }^{\circ} \mathrm{C}$ overnight and then poured on crushed ice. The solution was carefully made alkaline with $25 \%$ ammonia and then extracted with $\mathrm{CHCl}_{3}(3 \times 20 \mathrm{~mL})$. The combined extracts were dried on anhydrous $\mathrm{Na}_{2} \mathrm{SO}_{4}$ and filtered.

[4-(3-Methoxybenzylamino)phenyl]-p-tolyl-methanone (19). The residue, after evaporation of the solvent, was purified by column chromatography eluting with cyclohexane/EtOAc (80:20) to give pure 19 as a yellow powder (22\%); $R_{f}=0.52\left(\mathrm{CHCl}_{3}-\mathrm{MeOH}, 98: 2\right) ; \mathrm{mp} .123-124{ }^{\circ} \mathrm{C} ;{ }^{1} \mathrm{H}-\mathrm{NMR}(300 \mathrm{MHz}$, $\left.\mathrm{CDCl}_{3}\right) \delta 2.32(\mathrm{~s}, 3 \mathrm{H}), 3.78(\mathrm{~s}, 3 \mathrm{H}), 4.37(\mathrm{~s}, 2 \mathrm{H}), 4.65($ br s, $1 \mathrm{H}), 6.60(\mathrm{~d}, J=7.7 \mathrm{~Hz}, 2 \mathrm{H}), 6.80-6.94$ $(\mathrm{m}, 2 \mathrm{H}), 7.22-7.28(\mathrm{~m}, 4 \mathrm{H}), 7.62(\mathrm{~d}, J=7.2 \mathrm{~Hz}, 2 \mathrm{H}), 7.71(\mathrm{~d}, J=7.7 \mathrm{~Hz}, 2 \mathrm{H}) ;{ }^{13} \mathrm{C}-\mathrm{NMR}(300 \mathrm{MHz}$, $\left.\mathrm{CDCl}_{3}\right) \delta 21.6(1 \mathrm{C}), 47.7(1 \mathrm{C}), 55.3(1 \mathrm{C}), 111.6(2 \mathrm{C}), 112.8(1 \mathrm{C}), 113.1$ (1C), $119.6(1 \mathrm{C}), 126.9(1 \mathrm{C})$, 127.7 (2C), 129.9 (2C), 130.6 (1C), 132.9 (2C), 136.2 (1C), 140.0 (1C), 141.9 (1C), 151.7 (1C), 160.0 (1C), 195.1 (1C); IR (thin film) $\mathrm{cm}^{-1} 3356,3017,2963,1636,1593,1528,1468,1262,1150$; MS (ESI, positive mode) $\mathrm{m} / z[M+\mathrm{H}]^{+} 332.16451\left(\mathrm{C}_{22} \mathrm{H}_{22} \mathrm{NO}_{2}\right.$ requires 332.15723); Anal. Calcd for $\mathrm{C}_{22} \mathrm{H}_{21} \mathrm{NO}_{2}$ : C 79.73, H 6.39, N 4.23, found: C 79.68, H 6.41, N 4.21. 
[4-(4-tert-Butylbenzylamino)phenyl]-p-tolyl-methanone (20). The residue, after evaporation of the solvent, was purified by column chromatography eluting with cyclohexane/EtOAc $(85: 15)$ to give pure 20 as a yellow powder (23\%); $R_{f}=0.57\left(\mathrm{CHCl}_{3}-\mathrm{MeOH}, 98: 2\right) ; \mathrm{mp} .140-141{ }^{\circ} \mathrm{C} ;{ }^{1} \mathrm{H}-\mathrm{NMR}(300 \mathrm{MHz}$, $\left.\mathrm{CDCl}_{3}\right) \delta 1.23(\mathrm{~s}, 9 \mathrm{H}), 2.32(\mathrm{~s}, 3 \mathrm{H}), 3.83(\mathrm{~s}, 2 \mathrm{H}), 4.12($ br s, $1 \mathrm{H}), 6.65(\mathrm{~d}, J=8.6 \mathrm{~Hz}, 2 \mathrm{H}), 7.10(\mathrm{~d}$, $J=7.8 \mathrm{~Hz}, 2 \mathrm{H}), 7.16-7.30(\mathrm{~m}, 4 \mathrm{H}), 7.62-7.75(\mathrm{~m}, 4 \mathrm{H}) ;{ }^{13} \mathrm{C}-\mathrm{NMR}\left(300 \mathrm{MHz}, \mathrm{CDCl}_{3}\right) \delta 22.8(1 \mathrm{C})$, 29.5 (3C), 32.0 (1C), 39.1 (1C), 121.1 (1C), 123.7 (1C), 126.8 (1C), 128.3 (2C), 128.9 (2C), 129.0 (2C), 129.4 (2C), 130.2 (2C), 130.8 (1C), 132.9 (2C), 143.0 (1C), 150.7 (1C), 194.2 (1C); IR (thin film) $\mathrm{cm}^{-1} 3422,3337,3024,2963,1636,1586,1501,1439,1319$; MS (ESI, positive mode) $\mathrm{m} / \mathrm{z}$ $[M+\mathrm{H}]^{+} 358.21016\left(\mathrm{C}_{25} \mathrm{H}_{28} \mathrm{NO}\right.$ requires 358.20926); Anal. Calcd for $\mathrm{C}_{25} \mathrm{H}_{27} \mathrm{NO}: \mathrm{C}$ 83.99, $\mathrm{H} 7.61, \mathrm{~N}$ 3.92, found: C 83.87, H 7.58, N 3.97.

p-Tolyl-[4-(3-trifluoromethylbenzylamino)phenyl]-methanone (21). The residue, after evaporation of the solvent, was purified by column chromatography eluting with cyclohexane/EtOAc (80:20) to give pure 21 as a reddish-orange oil $(39 \%) ; R_{f}=0.70\left(\mathrm{CHCl}_{3}-\mathrm{MeOH}, 98: 2\right) ;{ }^{1} \mathrm{H}-\mathrm{NMR}\left(300 \mathrm{MHz}, \mathrm{CDCl}_{3}\right) \delta$ $2.38(\mathrm{~s}, 3 \mathrm{H}), 4.46(\mathrm{~s}, 2 \mathrm{H}), 4.78(\mathrm{br} \mathrm{s}, 1 \mathrm{H}), 6.58(\mathrm{~d}, J=5.2 \mathrm{~Hz}, 2 \mathrm{H}), 7.23(\mathrm{~d}, J=9.2 \mathrm{~Hz}, 2 \mathrm{H}), 7.43-7.74$ $(\mathrm{m}, 8 \mathrm{H}) ;{ }^{13} \mathrm{C}-\mathrm{NMR}\left(300 \mathrm{MHz}, \mathrm{CDCl}_{3}\right) \delta 21.5$ (1C), 47.3 (1C), 111.6 (2C), 111.8 (1C), 113.7 (1C), 125.1 (1C), 127.6 (1C), 128.6 (2C), 129.2 (2C), 129.8 (1C), 132.9 (2C), 134.3 (1C), 136.2 (1C), 138.8 (1C), 141.9 (1C), 143.5 (1C), 151.4 (1C), 195.1 (1C); IR (thin film) $\mathrm{cm}^{-1} 3356,3024,2924,1651$, 1593, 1527, 1439; MS (ESI, positive mode) $\mathrm{m} / z[M+\mathrm{H}]^{+} 370.14133\left(\mathrm{C}_{22} \mathrm{H}_{19} \mathrm{~F}_{3} \mathrm{NO}\right.$ requires 370.13405$)$.

p-Tolyl-[4-(4-trifluoromethylbenzylamino)phenyl]-methanone (22). The residue, after evaporation of the solvent, was purified by column chromatography eluting with cyclohexane/EtOAc (80:20) to give pure 22 as a dark-yellow powder $(96 \%) ; R_{f}=0.49\left(\mathrm{CHCl}_{3}-\mathrm{MeOH}, 98: 2\right) ;$ mp. $115-116{ }^{\circ} \mathrm{C} ;{ }^{1} \mathrm{H}-\mathrm{NMR}$ $\left(300 \mathrm{MHz} \mathrm{CDCl}_{3}\right) \delta 2.35(\mathrm{~s}, 3 \mathrm{H}), 4.49(\mathrm{~s}, 2 \mathrm{H}), 4.82(\mathrm{br} \mathrm{s}, 1 \mathrm{H}), 6.60(\mathrm{dd}, J=9.0,1.9 \mathrm{~Hz}, 2 \mathrm{H}), 7.27$ (t, $J=8.7 \mathrm{~Hz}, 2 \mathrm{H}), 7.42(\mathrm{~d}, J=6.2 \mathrm{~Hz}, 2 \mathrm{H}), 7.60(\mathrm{dd}, J=9.0,1.9 \mathrm{~Hz}, 2 \mathrm{H}), 7.66-7.78(\mathrm{~m}, 4 \mathrm{H}) ;{ }^{13} \mathrm{C}-\mathrm{NMR}$ $\left(300 \mathrm{MHz}, \mathrm{CDCl}_{3}\right) \delta 21.6(1 \mathrm{C}), 47.3$ (1C), 111.7 (2C), 127.0 (1C), 127.7 (1C), $128.8(2 \mathrm{C}), 129.4(2 \mathrm{C})$, 130.2 (2C), 130.5 (2C), 130.6 (1C), 130.7 (2C), 131.1 (1C), 132.9 (1C), 142.0 (1C), 152.0 (1C), 195.1 (1C); IR (thin film) $\mathrm{cm}^{-1} 3356,3021,2920,1647,1597,1528,1451$; MS (ESI, positive mode) $\mathrm{m} / \mathrm{z}$ $[M+\mathrm{H}]^{+} 370.14133\left(\mathrm{C}_{22} \mathrm{H}_{19} \mathrm{~F}_{3} \mathrm{NO}\right.$ requires 370.13405); Anal. Calcd for $\mathrm{C}_{22} \mathrm{H}_{18} \mathrm{~F}_{3} \mathrm{NO}: \mathrm{C} 71.53, \mathrm{H} 4.91$, N 3.79, found: C 71.48, H 4.95, N 3.67.

[4-(3-Methoxybenzylamino)phenyl]-(4-methoxyphenyl)-methanone (23). The residue, after evaporation of the solvent, was purified by column chromatography eluting with $\mathrm{CHCl}_{3} / \mathrm{MeOH}(99: 1)$ to give pure 23 as an orange oil (28\%); $R_{f}=0.44\left(\mathrm{CHCl}_{3}-\mathrm{MeOH}, 98: 2\right) ;{ }^{1} \mathrm{H}-\mathrm{NMR}\left(300 \mathrm{MHz}, \mathrm{CDCl}_{3}\right) \delta 3.73$ (s, $3 \mathrm{H}), 3.85(\mathrm{~s}, 3 \mathrm{H}), 4.37(\mathrm{~s}, 2 \mathrm{H}), 4.63(\mathrm{br} \mathrm{s}, 1 \mathrm{H}), 6.59(\mathrm{~d}, J=8.7 \mathrm{~Hz}, 2 \mathrm{H}), 6.78-6.95(\mathrm{~m}, 6 \mathrm{H}), 7.65-7.74$ $(\mathrm{m}, 4 \mathrm{H}) ;{ }^{13} \mathrm{C}-\mathrm{NMR}\left(300 \mathrm{MHz}, \mathrm{CDCl}_{3}\right) \delta 47.6(1 \mathrm{C}), 55.3(1 \mathrm{C}), 55.7$ (1C), $111.3(2 \mathrm{C}), 113.3(2 \mathrm{C})$, 120.3 (1C), 127.0 (1C), 129.0 (1C), 129.9 (1C), 130.8 (1C), 131.5 (1C), $132.0(2 \mathrm{C}), 132.8$ (2C), 140.1 (1C), 151.6 (1C), 160.0 (1C), 162.4 (1C), 194.3 (1C); IR (thin film) $\mathrm{cm}^{-1} 3352,3005,2925,1636$, 1597, 1528, 1458, 1316, 1258, 1169; MS (ESI, positive mode) $m / z[M+\mathrm{H}]^{+} 348.14725\left(\mathrm{C}_{22} \mathrm{H}_{22} \mathrm{NO}_{3}\right.$ requires 348.15214 ). 
[4-(4-tert-Butylbenzylamino)phenyl]-(4-methoxyphenyl)-methanone (24). The residue, after evaporation of the solvent, was purified by column chromatography eluting with cyclohexane/EtOAc (80:20) to give pure 24 as a red oil $(60 \%) ; R_{f}=0.52\left(\mathrm{CHCl}_{3}-\mathrm{MeOH}, 98: 2\right) ;{ }^{1} \mathrm{H}-\mathrm{NMR}(300 \mathrm{MHz}$, $\left.\mathrm{CDCl}_{3}\right) \delta 1.26(\mathrm{~s}, 9 \mathrm{H}), 3.84(\mathrm{~s}, 3 \mathrm{H}), 3.89(\mathrm{~s}, 2 \mathrm{H}), 4.10(\mathrm{br} \mathrm{s}, 1 \mathrm{H}), 6.65(\mathrm{~d}, J=8.2 \mathrm{~Hz}, 1 \mathrm{H}), 6.93(\mathrm{~d}$, $J=8.6 \mathrm{~Hz}, 2 \mathrm{H}), 7.11(\mathrm{~d}, J=8.2 \mathrm{~Hz}, 1 \mathrm{H}), 7.20(\mathrm{~d}, J=7.6 \mathrm{~Hz}, 2 \mathrm{H}), 7.29(\mathrm{t}, J=7.6 \mathrm{~Hz}, 2 \mathrm{H}), 7.58-7.66$ $(\mathrm{m}, 2 \mathrm{H}), 7.78(\mathrm{~d}, J=8.6 \mathrm{~Hz}, 2 \mathrm{H}) ;{ }^{13} \mathrm{C}-\mathrm{NMR}\left(300 \mathrm{MHz}, \mathrm{CDCl}_{3}\right) \delta 31.4(3 \mathrm{C}), 37.7(1 \mathrm{C}), 38.2(1 \mathrm{C}), 55.5$ (1C), 123.7 (2C), 125.7 (1C), 126.7 (1C), 128.1 (1C), 128.4 (2C), 128.9 (2C), 131.1 (1C), 131.4 (2C), 133.9 (1C), 135.4 (1C), 138.6 (1C), 149.2 (1C), 149.6 (1C), 162.5 (1C), 194.5 (1C); IR (thin film) $\mathrm{cm}^{-1}$ 3476, 3364, 3005, 2963, 1620, 1601, 1508, 1458, 1420, 1254; MS (ESI, positive mode) $\mathrm{m} / z[M+\mathrm{H}]^{+}$ $374.19873\left(\mathrm{C}_{25} \mathrm{H}_{28} \mathrm{NO}_{2}\right.$ requires 374.20418).

(4-Methoxy-phenyl)-[4-(3-trifluoromethyl-benzylamino)-phenyl]-methanone (25). The residue, after evaporation of the solvent, was purified by column chromatography eluting with cyclohexane/EtOAc $(80: 20)$ to give pure 25 as a red oil $(33 \%) ; R_{f}=0.60\left(\mathrm{CHCl}_{3}-\mathrm{MeOH}, 98: 2\right) ;{ }^{1} \mathrm{H}-\mathrm{NMR}(300 \mathrm{MHz}$, $\left.\mathrm{CDCl}_{3}\right) \delta 3.82(\mathrm{~s}, 3 \mathrm{H}), 4.46(\mathrm{~s}, 2 \mathrm{H}), 4.73(\mathrm{br} \mathrm{s}, 1 \mathrm{H}), 6.60(\mathrm{dd}, J=6.9,1.8 \mathrm{~Hz}, 2 \mathrm{H}), 6.93(\mathrm{dd}, J=6.9$, $1.8 \mathrm{~Hz}, 2 \mathrm{H}), 7.45-7.61(\mathrm{~m}, 4 \mathrm{H}), 7.64-7.74(\mathrm{~m}, 4 \mathrm{H}) ;{ }^{13} \mathrm{C}-\mathrm{NMR}\left(300 \mathrm{MHz}, \mathrm{CDCl}_{3}\right) \delta 47.2(1 \mathrm{C}), 55.5$ (1C), 111.7 (2C), 113.4 (2C), 120.4 (1C), 123.9 (1C), 124.4 (1C), 127.5 (1C), 129.0 (1C), 129.3 (1C), 130.6 (1C), 130.9 (1C), 131.4 (2C), 132.0 (2C), 139.7 (1C), 151.2 (1C), 162.5 (1C), 194.3 (1C); IR (thin film) $\mathrm{cm}^{-1} 3348,3032,2932,1636,1601,1528,1455,1327,1169$; MS (ESI, positive mode) $\mathrm{m} / \mathrm{z}$ $[M+\mathrm{H}]^{+} 386.13624\left(\mathrm{C}_{22} \mathrm{H}_{19} \mathrm{~F}_{3} \mathrm{NO}_{2}\right.$ requires 386.12896).

(4-Methoxyphenyl)-[4-(4-trifluoromethylbenzylamino)phenyl]-methanone (26). The residue, after evaporation of the solvent, was purified by column chromatography eluting with $\mathrm{CH}_{2} \mathrm{Cl}_{2} / \mathrm{EtOH}(98: 2)$ to give pure 26 as a yellow oil $(22 \%) ; R_{f}=0.56\left(\mathrm{CHCl}_{3}-\mathrm{MeOH}, 98: 2\right) ;{ }^{1} \mathrm{H}-\mathrm{NMR}\left(300 \mathrm{MHz}, \mathrm{CDCl}_{3}\right) \delta$ $3.71(\mathrm{~s}, 3 \mathrm{H}), 4.41(\mathrm{~s}, 2 \mathrm{H}), 4.70(\mathrm{br} \mathrm{s}, 1 \mathrm{H}), 6.50(\mathrm{dd}, J=9.5,4.0 \mathrm{~Hz}, 2 \mathrm{H}), 6.89(\mathrm{dd}, J=9.3,3.2 \mathrm{~Hz}, 2 \mathrm{H})$, $7.20(\mathrm{t}, J=5.0 \mathrm{~Hz}, 2 \mathrm{H}), 7.38(\mathrm{dd}, J=9.3,3.2 \mathrm{~Hz}, 2 \mathrm{H}), 7.52(\mathrm{t}, J=5.0 \mathrm{~Hz}, 2 \mathrm{H}), 7.64(\mathrm{dd}, J=9.5,4.0$ $\mathrm{Hz}, 2 \mathrm{H}) ;{ }^{13} \mathrm{C}-\mathrm{NMR}\left(300 \mathrm{MHz}, \mathrm{CDCl}_{3}\right) \delta 47.4(1 \mathrm{C}), 55.9(1 \mathrm{C}), 111.6(1 \mathrm{C}), 111.9(2 \mathrm{C}), 113.6(2 \mathrm{C})$, 122.5 (1C), 126.0 (2C), 127.7 (2C), 129.2 (1C), 131.5 (2C), 132.9 (2C), 142.8 (1C), 151.3 (1C), 157.1 (1C), 162.7 (1C), 194.4 (1C); IR (thin film) $\mathrm{cm}^{-1} 3345,3035,2963,1636,1597,1531,1462,1323$, 1165; MS (ESI, positive mode) $m / z[M+\mathrm{H}]^{+} 386.13624\left(\mathrm{C}_{22} \mathrm{H}_{19} \mathrm{~F}_{3} \mathrm{NO}_{2}\right.$ requires 386.12896).

(2,4-Dimethoxyphenyl)-[4-(3-methoxybenzylamino)phenyl]-methanone (27). The residue, after evaporation of the solvent, was purified by column chromatography eluting with cyclohexane/EtOAc (75:25) to give 27 pure as a pink powder (53\%); $R_{f}=0.33\left(\mathrm{CHCl}_{3}-\mathrm{MeOH}, 98: 2\right) ; \mathrm{mp} .126-127{ }^{\circ} \mathrm{C} ;{ }^{1} \mathrm{H}-$ NMR (300 MHz, $\left.\mathrm{CDCl}_{3}\right) \delta 3.68(\mathrm{~s}, 3 \mathrm{H}), 3.77(\mathrm{~s}, 3 \mathrm{H}), 3.83(\mathrm{~s}, 3 \mathrm{H}), 4.34(\mathrm{~s}, 2 \mathrm{H}), 4.66(\mathrm{br} \mathrm{s}, 1 \mathrm{H}), 6.48-$ $6.55(\mathrm{~m}, 4 \mathrm{H}), 6.78-6.91(\mathrm{~m}, 4 \mathrm{H}), 7.25(\mathrm{t}, J=7.7 \mathrm{~Hz}, 1 \mathrm{H}), 7.99(\mathrm{~d}, J=8.7 \mathrm{~Hz}, 2 \mathrm{H}) ;{ }^{13} \mathrm{C}-\mathrm{NMR}(300$ $\left.\mathrm{MHz}, \mathrm{CDCl}_{3}\right) \delta 47.6(1 \mathrm{C}), 55.3$ (1C), 55.5 (1C), 55.7 (1C), 98.6 (1C), $104.2(1 \mathrm{C}), 111.5(2 \mathrm{C}), 112.8$ (1C), 113.1 (1C), 119.6 (1C), 122.6 (1C), 127.7 (1C), 129.9 (1C), 131.1 (1C), 132.7 (2C), 140.1 (1C), 151.9 (1C), 158.8 (1C), 160.0 (1C), 162.3 (1C), 194.0 (1C); IR (thin film) $\mathrm{cm}^{-1} 3348,3005,2936$, 1636, 1597, 1489, 1458, 1316, 1262, 1161; MS (ESI, positive mode) $m / z[M+\mathrm{H}]^{+} 378.15691$ $\left(\mathrm{C}_{23} \mathrm{H}_{24} \mathrm{NO}_{4}\right.$ requires 378.16271); Anal. Calcd for $\mathrm{C}_{23} \mathrm{H}_{23} \mathrm{NO}_{4}: \mathrm{C} 73.19, \mathrm{H} \mathrm{6.14}, \mathrm{N} 3.71$, found: $\mathrm{C}$ $73.25, \mathrm{H} 6.21, \mathrm{~N} 3.68$. 
[4-(4-tert-Butylbenzylamino)phenyl]-(2,4-dimethoxyphenyl)-methanone (28). The residue, after evaporation of the solvent, was purified by column chromatography eluting with cyclohexane/EtOAc (80:20) to give pure 28 as a brown powder $(82 \%) ; R_{f}=0.45\left(\mathrm{CHCl}_{3}-\mathrm{MeOH}, 98: 2\right)$; mp. $138-139{ }^{\circ} \mathrm{C}$; ${ }^{1} \mathrm{H}-\mathrm{NMR}\left(300 \mathrm{MHz}, \mathrm{CDCl}_{3}\right) \delta 1.27$ (s, 9H), 3.70 (s, 3H), 3.77 (s, 3H), 3.89 (s, 2H), 4.08 (br s, 1H), $6.50(\mathrm{~d}, J=8.6 \mathrm{~Hz}, 2 \mathrm{H}), 6.58(\mathrm{~d}, J=8.3 \mathrm{~Hz}, 2 \mathrm{H}), 7.08(\mathrm{~d}, J=8.6 \mathrm{~Hz}, 2 \mathrm{H}), 7.24-7.31(\mathrm{~m}, 3 \mathrm{H}), 7.57(\mathrm{~d}$, $J=8.3 \mathrm{~Hz}, 1 \mathrm{H}), 7.64(\mathrm{~d}, J=5.5 \mathrm{~Hz}, 1 \mathrm{H}) ;{ }^{13} \mathrm{C}-\mathrm{NMR}\left(300 \mathrm{MHz}, \mathrm{CDCl}_{3}\right) \delta 31.4(3 \mathrm{C}), 34.5(1 \mathrm{C}), 37.6$ (1C), 55.5 (1C), 55.6 (1C), 98.8 (1C), 104.5 (1C), 114.5 (1C), 122.5 (1C), 125.7 (2C), 128.0 (2C), 128.8 (1C), 131.1 (1C), 131.3 (2C), 133.8 (2C), 135.5 (1C), 149.6 (1C), 158.9 (1C), 162.5 (1C), 194.4 (1C); IR (thin film) $\mathrm{cm}^{-1} 3480,3364,3005,2963,1623,1602,1505,1462,1410,1312,1277,1211$; MS (ESI, positive mode) $m / z[M+\mathrm{H}]^{+} 404.22034\left(\mathrm{C}_{26} \mathrm{H}_{30} \mathrm{NO}_{3}\right.$ requires 404.21474); Anal. Calcd for $\mathrm{C}_{26} \mathrm{H}_{29} \mathrm{NO}_{3}$ : C 77.39, H 7.24, N 3.47, found: C 77.42, H 7.30, N 3.51.

(2,4-Dimethoxyphenyl)-[4-(3-trifluoromethylbenzylamino)phenyl]-methanone (29). The residue, after evaporation of the solvent, was purified by column chromatography eluting with cyclohexane/EtOAc (75:25) to give pure 29 as an olive-green oil (32\%); $R_{f}=0.58\left(\mathrm{CHCl}_{3}-\mathrm{MeOH}, 98: 2\right) ;{ }^{1} \mathrm{H}-\mathrm{NMR}(300$ $\left.\mathrm{MHz}, \mathrm{CDCl}_{3}\right) \delta 3.71(\mathrm{~s}, 3 \mathrm{H}), 3.82(\mathrm{~s}, 3 \mathrm{H}), 4.43(\mathrm{~s}, 2 \mathrm{H}), 4.83$ (br s, 1H), 6.46-6.55 (m, 4H), 7.25 (dd, $J=7.1,2.0 \mathrm{~Hz}, 1 \mathrm{H}), 7.42-7.58(\mathrm{~m}, 4 \mathrm{H}), 7.67(\mathrm{~d}, J=8.8 \mathrm{~Hz}, 2 \mathrm{H}) ;{ }^{13} \mathrm{C}-\mathrm{NMR}\left(300 \mathrm{MHz}, \mathrm{CDCl}_{3}\right) \delta 47.2$ (1C), 55.5 (1C), 55.7 (1C), 98.9 (1C), 104.2 (1C), 111.6 (2C), 122.5 (1C), 123.9 (1C), 124.0 (1C), 124.3 (1C), 124.4 (1C), 128.1 (1C), 129.3 (1C), 130.6 (1C), 131.1 (1C), 132.6 (2C), 139.7 (1C), 151.6 (1C), 158.9 (1C), 162.4 (1C), 194.1 (1C); IR (thin film) $\mathrm{cm}^{-1} 3337,3009,2936,1636,1597,1528$, 1505, 1458, 1327, 1161; MS (ESI, positive mode) $m / z[M+\mathrm{H}]^{+} 416.14680\left(\mathrm{C}_{23} \mathrm{H}_{21} \mathrm{~F}_{3} \mathrm{NO}_{3}\right.$ requires 416.13953).

(2,4-Dimethoxyphenyl)-[4-(4-trifluoromethylbenzylamino)phenyl]-methanone (30). The residue, after evaporation of the solvent, was purified by column chromatography eluting with cyclohexane/EtOAc (75:25) to give pure 30 as a dark-brown oil (50\%); $R_{f}=0.44\left(\mathrm{CHCl}_{3}-\mathrm{MeOH}, 98: 2\right) ;{ }^{1} \mathrm{H}-\mathrm{NMR}(300$ $\left.\mathrm{MHz}, \mathrm{CDCl}_{3}\right) \delta 3.72(\mathrm{~s}, 3 \mathrm{H}), 3.82(\mathrm{~s}, 3 \mathrm{H}), 4.45(\mathrm{~s}, 2 \mathrm{H}), 4.76($ br s, $1 \mathrm{H}), 6.48(\mathrm{dd}, J=7.9,1.9 \mathrm{~Hz}, 2 \mathrm{H})$, $6.53(\mathrm{~d}, J=8.0 \mathrm{~Hz}, 2 \mathrm{H}), 7.26(\mathrm{~d}, J=2.9 \mathrm{~Hz}, 1 \mathrm{H}), 7.45(\mathrm{~d}, J=8.1 \mathrm{~Hz}, 2 \mathrm{H}), 7.57(\mathrm{~d}, J=8.1 \mathrm{~Hz}, 2 \mathrm{H})$, $7.66(\mathrm{dd}, J=7.9,1.9 \mathrm{~Hz}, 2 \mathrm{H}) ;{ }^{13} \mathrm{C}-\mathrm{NMR}\left(300 \mathrm{MHz}, \mathrm{CDCl}_{3}\right) \delta 47.1$ (1C), 55.5 (1C), 55.7 (1C), 98.5 (1C), 98.9 (1C), 104.4 (1C), 111.6 (2C), 122.5 (1C), 125.6 (1C), 125.8 (2C), 127.4 (2C), 128.2 (1C), 129.6 (1C), 132.6 (2C), 142.7 (1C), 151.5 (1C), 158.9 (1C), 162.4 (1C), 194.0 (1C); IR (thin film) $\mathrm{cm}^{-1}$ $3345,3009,2940,1636,1597,1528,1505,1462$, 1323, 1161; MS (ESI, positive mode) $\mathrm{m} / z[M+\mathrm{H}]^{+}$ $416.14680\left(\mathrm{C}_{23} \mathrm{H}_{21} \mathrm{~F}_{3} \mathrm{NO}_{3}\right.$ requires 416.13953).

\section{CETP inhibition assay}

CETP inhibitory bioactivities were assayed by fluorescent-CE transfer employing commercially available kit (BioVision, Mountain View, CA, USA). The assay kit is based on donor molecule containing fluorescent self-quenched neutral lipid that is transferred to an acceptor molecule in the presence of CETP (from rabbit serum). CETP-mediated transfer of the fluorescent neutral lipid to the acceptor molecule results in increase in fluorescence. Inhibition of CETP will prevent lipid transfer and therefore decrease fluorescence intensity. 
The assay procedure can be described briefly as follows: an aliquot of rabbit serum $(1.5 \mu \mathrm{L})$ was added to testing sample $(160 \mu \mathrm{L})$. Then the master mix, provided in the assay kit (donor molecule, acceptor molecule and assay buffer, $20 \mu \mathrm{L}$ ) was added, mixed well, and the volume was completed to $203 \mu \mathrm{L}$ with the provided assay buffer. After incubation at $37{ }^{\circ} \mathrm{C}$ for 1 hour, fluorescence intensity (Excitation $\lambda$ : $465 \mathrm{~nm}$; Emission $\lambda$ : $535 \mathrm{~nm}$ ) was read in a FLX800TBI Microplate Fluorimeter (BioTek Instruments, Winooski, VT, USA).

The tested compounds were initially dissolved in DMSO to yield $10 \mathrm{mM}$ stock solutions and subsequently diluted to the required concentrations using distilled deionized water. The final concentration of DMSO was adjusted to $0.1 \%$. The percentage of residual activity of CETP was determined for each compound by comparing the activity of CETP in the presence and absence of the tested compound. Positive controls were tested to assess the degree of CETP inhibition by $0.1 \%$ DMSO. CETP was not affected by DMSO. Negative controls lacking rabbit serum were used as background. All measurements were conducted in duplicates.

\section{Acknowledgments}

We are grateful to the Scientific Research and Postgraduate Deanship at Al-Zaytoonah Private University of Jordan for sponsoring this project.

\section{References}

1. Hansson, G.K. Inflammation, atherosclerosis, and coronary artery disease. N. Engl. J. Med. 2005, $352,1685-1695$.

2. Lamarche, B.; Despres, J.P.; Moorjani, S.; Cantin, B.; Dagenais, G.R.; Lupien, P.J. Triglycerides and HDL-cholesterol as risk factors for ischemic heart disease, Results from the Quebec cardiovascular study. Atherosclerosis 1996, 119, 235-245.

3. Tall, A.R.; Wang, N.; Mucksavage, P. Is it time to modify the reverse cholesterol transport model? J. Clin. Invest. 2001, 108, 1273-1275.

4. Qiu, X.; Mistry, A.; Ammirati, M.J.; Chrunyk, B.A.; Clark, R.W.; Cong, Y.; Culp, J.S.; Danley, T.B.; Freeman, K.F.; Geoghegan, M.C.; et al. Crystal structure of cholesteryl ester transfer protein reveals a long tunnel and four bound lipid molecules. Nat. Struct. Mol. Biol. 2007, 14, 106-113.

5. Boekholdt, S.M.; Kuivenhoven, J.A.; Wareham, N.J.; Peters, R.J.G.; Jukema, J.W.; Luben, R.; Bingham, S.A.; Day, N.E.; Kastelein, J.J.P.; Khaw, K.T. Plasma levels of cholesteryl ester transfer protein and the risk of future coronary artery disease in apparently healthy men and women. Circulation 2004, 110, 1418-1423.

6. McPherson, R.; Mann, C.J.; Tall, A.R.; Hogue, M.; Martin, L.; Milne, R.W.; Marcel, Y.L. Plasma concentration of cholesteryl ester transfer protein in hyperlipoproteinemia. Arterioscler. Thromb. 1991, 11, 797-804.

7. Castilho; Marcelo, S.; Guido; Rafael, V.C.; Andricopulo; Adriano, D. 2D Quantitative structureactivity relationship studies on a series of cholesteryl ester transfer protein inhibitors. Bioorg. Med. Chem. 2007, 15, 6242-6252.

8. Hanumantharao, P.; Sambasivarao, S.V.; Soni, L.K.; Gupta, A.K.; Kaskhedikar, S.G. QSAR analysis of analogs of bis[2-(acylamino) $\mathrm{Ph}$ ] disulfides, 2-(acylamino) benzenethiols and S-[2- 
(acylamino) $\mathrm{Ph}$ ] alkanethioates as antihyperlipidemic agents. Indian J. Chem. 2005, 44B, 14811486.

9. Kelkar; Mandar, A.; Pednekar; Deepa, V.; Pimple; Surekha, R.; Akamanchi; Krishnacharya, G. 3D QSAR studies of inhibitors of cholesterol ester transfer protein (CETP) by CoMFA, CoMSIA and GFA methodologies. Med. Chem. Res. 2004, 13, 590-604.

10. Cronin, M.T.D.; Schultz, T.W. Pitfalls in QSAR. J. Mol. Struct. 2003, 622, 39-51.

11. Akamatsu, M. Current state and perspectives of 3D-QSAR. Curr. Top. Med. Chem. 2002, 12, 1381-1394.

12. Abu Khalaf, R.; Abu Sheikha, G.; Bustanji, Y.; Taha, M.O. Discovery of new cholesteryl ester transfer protein inhibitors via ligand-based pharmacophore modeling and QSAR analysis followed by synthetic exploration. Eur. J. Med. Chem. 2010, 45, 1598-1617.

13. Carey, F.A., Sundberg, R.J. Advanced Organic Chemistry, $5^{\text {th }}$ Ed.; Springer: New York, 2008; Vol. A.

14. del Amo, V.; Philp, D. Making imines without making water-exploiting a recognition-mediated Aza-Wittig reaction. Org. Lett. 2009, 11, 301-304.

15. Ivanov, I.; Nikolova, S.; Statkova-Abeghe, S. Efficient one-pot friedel-crafts acylation of benzene and its derivatives with unprotected aminocarboxylic acids in polyphosphoric acid. Synth. Commun. 2006, 36, 1405-1411.

16. Reinhard, E.J.; Wang, J.L.; Durley, R.C.; Fobian, Y.M.; Grapperhaus, M.L.; Hickory, M.A.; Massa, M.B.; Norton, M.A.; Promo, M.B.; Tollefson, W.F.; et al. Discovery of a simple picomolar inhibitor of cholesteryl ester transfer protein. J. Med. Chem. 2003, 46, 2152-2168.

Sample Availability: Not available.

(C) 2010 by the authors; licensee MDPI, Basel, Switzerland. This article is an Open Access article distributed under the terms and conditions of the Creative Commons Attribution license (http://creativecommons.org/licenses/by/3.0/). 